\title{
Equilibrium statistical mechanics of a grain boundary
}

\author{
D. B. Abraham, ${ }^{1,2}$ Ville Mustonen, ${ }^{1,2}$ and A. J. Wood ${ }^{1,3}$ \\ ${ }^{1}$ Theoretical Physics, University of Oxford, Oxford OX1 3NP, United Kingdom \\ ${ }^{2}$ Laboratory of Computational Engineering, Helsinki University of Technology, P.O. Box 9203, FIN-02015, Finland \\ ${ }^{3}$ School of Physics, James Clerk Maxwell Building, University of Edinburgh, Edinburgh, EH9 3JZ, United Kingdom
}

(Received 16 October 2004; published 8 March 2005)

\begin{abstract}
In this article we introduce, develop, and discuss the theoretical calculations required for the exact solution of a recently reported phase transition, the geodesic to zigzag transition. In this scenario the interfacial transition emerges from geometric competition between a geodesic, shortest path, configuration and a zigzag configuration which is able to reduce its energy by binding to a centrally positioned defect line. From a technical point of view the transition is unusual as it is described by a change from saddle dominated behavior to pole dominated behavior of the integral representing the partition function ratio. We also establish the precise fluctuation behavior of the interface by computing the spin magnetization at any point in the system.
\end{abstract}

DOI: 10.1103/PhysRevE.71.036106

PACS number(s): 64.60.-i, 68.08.Bc, 68.35.Rh

\section{INTRODUCTION}

Considerable insight into the wetting behavior near surfaces in low dimensions has been gained by understanding the delocalization transitions of interfaces within the twodimensional Ising model. This model is a well known statistical mechanical problem that may be solved exactly with well established techniques of mathematical physics [1-3]. Interfacial results may be derived by considering, in magnetic language, the behavior of domain walls between the positive and negative phases. With the lattice gas interpretation we can then interpret these domain walls as interfaces separating a gas phase from a fluid phase, or, in terms of mixtures, with coexisting phases geometrically controlled by differential surface fugacities. The relevance of geometry for surface phase transitions is a matter of considerable current interest [4-7]. As the solutions are exact, they incorporate all possible fluctuations of the interface. The use of the Ising model analogy has made it possible to categorize approximation schemes in a systematic way, permitting greater understanding of situations which are beyond the scope of the Ising solution [8].

We may induce controlled interfacial configurations in a ferromagnetic model by flipping sequences of spins on the boundaries, thus forming differing regions of positive phase, negative phase, and the necessary domain walls. Energetic "traps" for the interface may also be introduced by weakening the bond strength of a particular line of bonds on the lattice. If this line runs perpendicular to the transfer direction, then an exact solution is still possible. A strip geometry, periodic horizontally, but with hard boundaries top and bottom, may be solved by transferring perpendicular to the walls. In such a geometry the possible interfacial behaviors are well understood.

If we position the line of weakened bonds adjacent to the wall then the well established delocalization or wetting transition occurs at a well defined temperature $T_{w}<T_{c}$ [9]. The transition mechanism here is produced by the basic energyentropy paradigm: the interface will gain energy by follow- ing the line of defect bonds, but will lose entropy as a result of the one-sided pinning against an attractive hard boundary. If, however, the "trap" is positioned centrally, in the thermodynamic limit, then the interface will be captured for all temperatures [10]. The differentiation between one-sided and two-sided pinning was highlighted by Fisher in a mesoscopic random walk model which indicates the generality of the scenario by careful analysis of the first return probabilities to lines [11]. Other work has considered the effect of boundary conditions that force the interface to cross the strip at a predetermined angle $[12,13]$ without the bond weakening. The results here are nontrivial because the surface tension associated with each unit length of interface crossing the lattice is manifestly angle dependent [12]. By introducing the bond weakening once more, next to the wall, it is possible to use the exact solution to define the contact angle in two dimensions in a way that is not invalidated by capillary fluctuations of the interface $[13,14]$.

Here we consider the natural remaining case in this scenario, namely, the behavior of an interface crossing the strip at an angle when a centrally positioned defect line, running parallel to the strip axis, is present. This introduces a competitive mechanism between the angled, geodesic interface configuration and one that takes a zigzag path across the system in order to profit from the line of weakened bonds. We have thus called this transition the geodesic to zigzag, or GZZ, transition. In this article we present the analytic details of this calculation including the computation of the magnetization profiles. Elsewhere we have presented a summary of these results [15], an approximation method [16], and a more detailed study of the relaxation dynamics [17] of the system.

\section{THE SYSTEM}

The system studied is depicted in Fig 1. We construct a ferromagnetic Ising system with identical hard wall boundary conditions $(h=+1)$ on the top and bottom but which is periodic along the horizontal axis. The lattice is anisotropic 


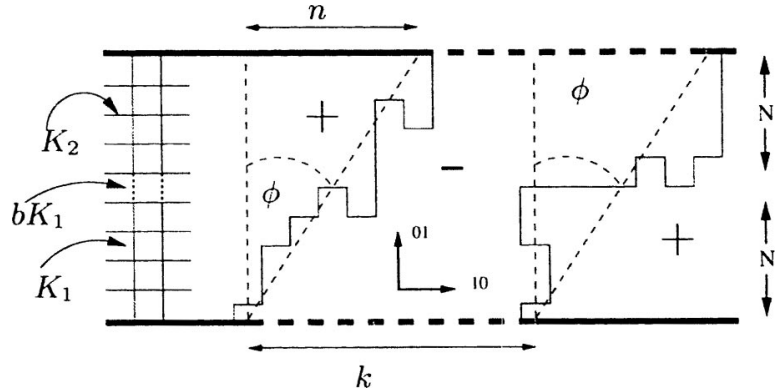

FIG. 1. A simple schematic diagram of the Ising system. We have shown the various bond couplings associated with the lattice. The boundary conditions are fixed to be plus on both the top and the bottom and thus the spin flips between the annotated spins induce a section of negative spins. Two possible configurations are shown. The one on the right would be a possible bound configuration along the center line whereas on the left is a diagonal, geodesic, configuration.

with a bond coupling of $K_{1}\left(K_{2}\right)$ between spins in a vertical (horizontal) direction. We choose the vertical distance to be $2 N+1$ and the system width to be $M$. We weaken the bonds in the $(N+1)$ th tier by a factor $b$; this represents the grain boundary. The boundary conditions are introduced by flipping a finite number $k$ of spins in both the top and the bottom tiers. We introduce the angle by offsetting the flips by $n$ spins. These boundary conditions, when we take appropriate limits, induce two - essentially identical - interfaces to form across the strip. We will demonstrate that the two most likely configurations are an interface crossing the strip at a mean angle $\phi$ or one which is partially pinned to the weakened tier of bonds in the center of the strip (see Fig. 1).

It is necessary to form two interfaces in the strip so that we may impose the periodic boundary conditions; for simplicity, the reader may prefer to visualize a single interface in a system with antiperiodic boundary conditions. Such a system is of course easier to simulate on a computer and is thus precisely what we have presented elsewhere, but this system is not feasible to treat analytically with the methods presented below. The disadvantage of introducing two interfaces into the system is that alternative, unwanted configurations may form. It is a feature of our exact treatment that we may precisely identify and thus eliminate such configurations. We can thus form simple closed expressions for the relevant interfacial terms.

\section{ISING COMPUTATION I: THE PHASE BOUNDARY}

The system described above can be solved exactly by constructing a transfer problem and transferring perpendicular to the hard boundaries. Denoting a line of spin flips between spins at $i$ and $j$ as $R(i, j)$ and the transfer matrices associated with the vertical and horizontal bonds as $V_{1}\left(K_{1}\right)$ and $V_{2}\left(K_{2}\right)$, respectively, we can write the partition function associated with a single interface, $Z(N, M ; b, \phi)$, in terms of matrix elements as
$Z(N, M ; b, \phi)^{2}$

$$
\begin{aligned}
& =\lim _{k \rightarrow \infty M \rightarrow \infty} \frac{\left\langle+\left|R(1, k)\left(V_{1} V_{2}\right)^{N} V_{b}\left(V_{2} V_{1}\right)^{N} R(n, n+k)\right|+\right\rangle}{\left\langle+\left|\left(V_{1} V_{2}\right)^{N} V_{b}\left(V_{2} V_{1}\right)^{N}\right|+\right\rangle} \\
& =\lim _{k \rightarrow \infty M \rightarrow \infty} e^{-2 K_{2}} \frac{\langle+|R(1, k) L R(n, n+k)|+\rangle}{\langle+|L|+\rangle}
\end{aligned}
$$

where

$$
L=V^{N} V_{2}^{1 / 2} V_{b} V_{2}^{1 / 2} V^{N}
$$

and

$$
V_{b} \equiv V_{1}\left(b K_{1}\right)=\exp -\sum_{1}^{M}\left(b K_{1}\right)^{*} \sigma_{j}^{z}
$$

for $0<b<1$ and the limiting process used in Eq. (2) will be described as we proceed. The relationship $R(i, j)|+\rangle=-\left(f_{i}^{\dagger}\right.$ $\left.+f_{i}\right)\left(f_{j}^{\dagger}+f_{j}\right)|+\rangle$ which is valid when $R(i, j)$ operates on $|+\rangle$ is useful in evaluating Eq. (2) if we bring in the identity

$$
| \pm\rangle=\frac{1}{\sqrt{2}}\left(\left|\Phi_{+}^{0}\right\rangle \pm\left|\Phi_{-}^{0}\right\rangle\right)
$$

where $\left|\Phi_{ \pm}^{0}\right\rangle$ are the simultaneous maximal eigenvectors of $V_{2}$ (note the strict degeneracies) and of the parity operator

$$
P_{M}=\prod_{j=1}^{M}\left(-\sigma_{j}^{z}\right)
$$

with

$$
P_{M}\left|\Phi_{ \pm}^{0}\right\rangle= \pm\left|\Phi_{ \pm}^{0}\right\rangle .
$$

Thus the matrix element in the numerator is the sum of the two terms $\left\langle\Phi_{ \pm}^{0}|R(1, k) L R(n, n+k)| \Phi_{ \pm}^{0}\right\rangle$. Since we are interested in the limit $M \rightarrow \infty$, in which the + and - terms are asymptotically identical, we will just display one term in the analysis that follows.

Expanding the local Fermi operators $f_{j}$ and their adjoints in terms of the $G_{0}^{\dagger}(\omega), G_{0}(-\omega)$ basis, defined by

$$
V_{2}(+) G_{0}^{\dagger}(\omega)\left|\Phi_{+}^{0}\right\rangle=e^{-2 K_{2}} G_{0}^{\dagger}(\omega)\left|\Phi_{+}^{0}\right\rangle
$$

independent of $\omega$, provided $e^{i M \omega}=-1$, which also defines the $G_{0}(\omega)$ which are related to the local fermions $f_{m}, f_{m}^{\dagger}$ by

$$
\left(\begin{array}{c}
G_{0}^{\dagger}(\omega) \\
G_{0}(-\omega)
\end{array}\right)=\left(\begin{array}{cc}
\cos \theta_{0}(\omega) & -i \sin \theta_{0}(\omega) \\
-i \sin \theta_{0}(\omega) & \cos \theta_{0}(\omega)
\end{array}\right)\left(\begin{array}{c}
F^{\dagger}(\omega) \\
F(-\omega)
\end{array}\right)
$$

with $\theta_{0}(\omega)=(\pi+\omega) / 2 \bmod \pi$, and

$$
F^{\dagger}(\omega)=\frac{1}{\sqrt{M}} \sum_{j=1}^{M} e^{i j \omega} f_{j}^{\dagger} .
$$

The transformation in (9), of the Bogolubov-Valatin type [18], requires the $G_{0}(\omega)$ to be Fermi operators, as are the $F(\omega)$ themselves. Simple algebra gives 


$$
\begin{aligned}
\left\langle\Phi_{+}^{0}|R(1, k) L R(n, n+k)| \Phi_{+}^{0}\right\rangle= & \frac{1}{M^{2}} \sum_{(\omega)_{4}} \exp \left(-i \omega_{1}\right) \exp \left[-i \omega_{2}(1+k)\right] \exp \left[-i \omega_{3}(1+n)\right] \exp \left[-i \omega_{4}(1+n+k)\right] \exp \left(i \sum_{1}^{4} \theta_{0}\left(\omega_{j}\right)\right) \\
& \times\left\langle\Phi_{+}^{0}\left|\left[G_{0}^{\dagger}\left(\omega_{1}\right)+G_{0}\left(-\omega_{1}\right)\right]\left[G_{0}^{\dagger}\left(\omega_{2}\right)+G_{0}\left(-\omega_{2}\right)\right] L\left[G_{0}^{\dagger}\left(\omega_{3}\right)+G_{0}\left(-\omega_{3}\right)\right]\left[G_{0}^{\dagger}\left(\omega_{4}\right)+G_{0}\left(-\omega_{4}\right)\right]\right| \Phi_{+}^{0}\right\rangle
\end{aligned}
$$

The matrix element in Eq. (11) can be evaluated by the following technique. Let $X^{\dagger}(\omega)$ be defined by the left vacuum relation

$$
\left\langle\Phi_{+}^{0}\right| L X^{\dagger}(\omega)=0
$$

and thus

$$
\left(\begin{array}{c}
X^{\dagger}(\omega) \\
Y(-\omega)
\end{array}\right)=L^{-1}\left(\begin{array}{c}
G_{0}^{\dagger}(\omega) \\
G_{0}(-\omega)
\end{array}\right) L
$$

where it is useful to introduce the auxiliary $Y(\omega)$ operators. The matrix element in Eq. (11) is then

$$
\begin{aligned}
\left\langle\Phi_{+}^{0}\right| & L\left[X^{\dagger}\left(\omega_{1}\right)+Y\left(-\omega_{1}\right)\right]\left[X^{\dagger}\left(\omega_{2}\right)+Y\left(-\omega_{2}\right)\right] \\
& \times\left[G_{0}^{\dagger}\left(\omega_{3}\right)+G_{0}\left(-\omega_{3}\right)\right]\left[G_{0}^{\dagger}\left(\omega_{4}\right)+G_{0}\left(-\omega_{4}\right)\right]\left|\Phi_{+}^{0}\right\rangle .
\end{aligned}
$$

An element of this form can be computed by a straightforward extension of standard methods, generalizing Wick's theorem to the case of asymmetric in and out states, which is useful in the present context. Consider the matrix element defined by

$$
\mathcal{M}\left((\omega)_{2 m}\right)=\left\langle a\left|\mathcal{O}_{1}\left(\omega_{1}\right) \cdots \mathcal{O}_{2 m}\left(\omega_{2 m}\right)\right| b\right\rangle
$$

where the in and out states $\langle a|$ and $|b\rangle$ have vacuum properties

$$
\langle a| X^{\dagger}(\omega)=0
$$

and

$$
Z(-\omega)|b\rangle=0
$$

together with the decomposition

$$
\mathcal{O}_{j}(\omega)=\alpha_{j}(\omega) X^{\dagger}(\omega)+\beta_{j}(\omega) Z(-\omega) .
$$

Note that this may not be possible in all circumstances. Using the definition (15) then by expanding $\mathcal{O}_{1}\left(\omega_{1}\right)$ using (18) and introducing the anticommutation relations $\left[Z\left(-\omega_{1}\right), \mathcal{O}_{j}\left(\omega_{j}\right)\right]_{+}$, which are $c$ numbers in this application, and the vacuum relations give

$$
\begin{aligned}
& \mathcal{M}\left((\omega)_{2 m}\right) \\
& \quad=\beta\left(\omega_{1}\right) \sum_{j=2}^{2 m}(-1)^{j}\left[Z\left(-\omega_{1}\right), \mathcal{O}_{j}\left(\omega_{j}\right)\right]_{+} \mathcal{M}\left(\Delta_{1} \Delta_{j}(\omega)_{2 m}\right)
\end{aligned}
$$

where $(\omega)_{2 m}=\left(\omega_{1}, \ldots, \omega_{2 m}\right)$ and $\Delta_{j}(\omega)_{I}=(\omega)_{I / j}$ if $j \in I$ for any index of the set $I$. Finally, we bring the anticommutator to a convenient form. Since it is a $c$ number, it follows that

$$
\left[Z\left(-\omega_{1}\right), \mathcal{O}_{j}\left(\omega_{j}\right)\right]_{+}=\frac{\left\langle a\left|\left[Z\left(-\omega_{1}\right), \mathcal{O}_{j}\left(\omega_{j}\right)\right]_{+}\right| b\right\rangle}{\langle a \mid b\rangle} .
$$

Using the vacuum relationship (17) leaves

$$
\left\langle a\left|Z\left(-\omega_{1}\right), \mathcal{O}_{j}\left(\omega_{j}\right)\right| b\right\rangle
$$

in the numerator; eliminating $Z\left(-\omega_{1}\right)$ using Eq. (18) and the vacuum relation (16) gives

$$
\left[Z\left(-\omega_{1}\right), \mathcal{O}_{j}\left(\omega_{j}\right)\right]_{+}=\frac{\left\langle a\left|\mathcal{O}_{1}\left(\omega_{1}\right) \mathcal{O}_{j}\left(\omega_{j}\right)\right| b\right\rangle}{\langle a \mid b\rangle}
$$

which is a very useful form for calculating, once the necessary conditions above have been established. As an example consider

$$
\begin{aligned}
M_{13}= & \left\langle\Phi_{+}^{0}\left|L\left[X^{\dagger}\left(\omega_{1}\right)+Y\left(-\omega_{1}\right)\right]\left[G_{0}^{\dagger}\left(\omega_{3}\right)+G_{0}\left(-\omega_{3}\right)\right]\right| \Phi_{+}^{0}\right\rangle \\
= & \left\langle\Phi_{+}^{0}\left|L Y\left(-\omega_{1}\right) G_{0}^{\dagger}\left(\omega_{3}\right)\right| \Phi_{+}^{0}\right\rangle=C\left(\omega_{1}\right) \\
& \times\left\langle\Phi_{+}^{0}\left|L G_{0}^{\dagger}\left(\omega_{1}\right) G_{0}^{\dagger}\left(\omega_{3}\right)\right| \Phi_{+}^{0}\right\rangle+D\left(\omega_{1}\right) \delta_{\omega_{1}, \omega_{3}}\left\langle\Phi_{+}^{0}|L| \Phi_{+}^{0}\right\rangle
\end{aligned}
$$

where the left and right vacuum operators are related by the unitary matrix transformation

$$
\left(\begin{array}{c}
X^{\dagger}(\omega) \\
Y(-\omega)
\end{array}\right)=\left(\begin{array}{ll}
A(\omega) & B(\omega) \\
C(\omega) & D(\omega)
\end{array}\right)\left(\begin{array}{c}
G_{0}^{\dagger}(\omega) \\
G_{0}(-\omega)
\end{array}\right),
$$

where the elements are to be computed in a manner demonstrated below. But, Eq. (16) also implies

$$
\begin{aligned}
0= & \left\langle\Phi_{+}^{0}\left|L X^{\dagger}\left(\omega_{1}\right) G_{0}^{\dagger}\left(\omega_{3}\right)\right| \Phi_{+}^{0}\right\rangle \\
= & A\left(\omega_{1}\right)\left\langle\Phi_{+}^{0}\left|L G_{0}^{\dagger}\left(\omega_{1}\right) G_{0}^{\dagger}\left(\omega_{3}\right)\right| \Phi_{+}^{0}\right\rangle \\
& +B\left(\omega_{1}\right) \delta_{\omega_{1},-\omega_{3}}\left\langle\Phi_{+}^{0}|L| \Phi_{+}^{0}\right\rangle .
\end{aligned}
$$

So, provided $A\left(\omega_{1}\right) \neq 0$,

$$
\frac{\left\langle\Phi_{+}^{0}\left|L G_{0}^{\dagger}\left(\omega_{1}\right) G_{0}^{\dagger}\left(\omega_{3}\right)\right| \Phi_{+}^{0}\right\rangle}{\left\langle\Phi_{+}^{0}|L| \Phi_{+}^{0}\right\rangle}=-\frac{B\left(\omega_{1}\right)}{A\left(\omega_{1}\right)} \delta_{\omega_{1},-\omega_{3}}
$$

and, finally,

$$
M_{13}=\left(D\left(\omega_{1}\right)-\frac{B\left(\omega_{1}\right) C\left(\omega_{1}\right)}{A\left(\omega_{1}\right)}\right) \delta_{\omega_{1},-\omega_{3}}\left\langle\Phi_{+}^{0}|L| \Phi_{+}^{0}\right\rangle
$$

which, by the unimodularity of Eq. (23), simplifies to

$$
M_{13}=\frac{1}{A\left(\omega_{1}\right)} \delta_{\omega_{1},-\omega_{3}}
$$

and the other contractions needed in this calculation follow in an analogous way. 
We now consider the calculation of the transformation matrix (23) in this particular example. It is useful to note the group property of the Bogoliubov-Valatin transformation (BVT) matrices. If

$$
U(\theta)=\left(\begin{array}{cc}
\cos \theta & -i \sin \theta \\
-i \sin \theta & \cos \theta
\end{array}\right),
$$

then $U\left(\theta_{1}+\theta_{2}\right)=U\left(\theta_{1}\right) U\left(\theta_{2}\right)$ and thus we have

$$
\begin{aligned}
V^{-N}\left(\begin{array}{c}
G_{0}^{\dagger}(\omega) \\
G_{0}(-\omega)
\end{array}\right) V^{N}= & U\left(\theta_{0}-\theta\right)\left(\begin{array}{cc}
e^{N \gamma(\omega)} & 0 \\
0 & e^{-N \gamma(\omega)}
\end{array}\right) U\left(\theta-\theta_{1}\right) \\
& \times\left(\begin{array}{c}
G_{1}^{\dagger}(\omega) \\
G_{1}(-\omega)
\end{array}\right)
\end{aligned}
$$

where the $G_{1}$ are the "diagonal" operators for the grainboundary modified matrix $V^{(b)} \equiv V_{2}^{1 / 2} V_{b} V_{2}^{1 / 2}$, with angle $\theta_{1}(\omega)$ in the BVT. This arrangement makes it easy to see that

$$
\begin{aligned}
L^{-1}\left(\begin{array}{c}
G_{0}^{\dagger}(\omega) \\
G_{0}(-\omega)
\end{array}\right) L= & U\left(\theta_{0}-\theta\right)\left(\begin{array}{cc}
e^{N \gamma(\omega)} & 0 \\
0 & e^{-N \gamma(\omega)}
\end{array}\right) U\left(\theta-\theta_{1}\right) \\
& \times\left(\begin{array}{cc}
e^{\gamma_{1}(\omega)} & 0 \\
0 & e^{-\gamma_{1}(\omega)}
\end{array}\right) U\left(\theta_{1}-\theta\right) \\
& \times\left(\begin{array}{cc}
e^{N \gamma(\omega)} & 0 \\
0 & e^{-N \gamma(\omega)}
\end{array}\right) U\left(\theta-\theta_{0}\right)\left(\begin{array}{c}
G_{0}^{\dagger}(\omega) \\
G_{0}(-\omega)
\end{array}\right) .
\end{aligned}
$$

Thus we have a mild exercise with $2 \times 2$ matrices or Pauli spin algebra according to taste. It is apparent that finite $N$ effects are obtained exactly, but, for the purposes of the present application, we will work to the leading asymptotic order. Thus Eq. (30) becomes

$$
L^{-1}\left(\begin{array}{c}
G_{0}^{\dagger}(\omega) \\
G_{0}(-\omega)
\end{array}\right) L \rightarrow e^{2 N \gamma(\omega)} Z_{\infty}(\omega)\left(\begin{array}{c}
G_{0}^{\dagger}(\omega) \\
G_{0}(-\omega)
\end{array}\right)
$$

with

$$
\begin{aligned}
Z_{\infty}(\omega)= & {\left[\cosh \gamma_{1}+\sinh \gamma_{1} \cos \left(\delta^{*}-\delta_{1}^{*}\right)\right] } \\
& \times \frac{1}{2}\left(\begin{array}{cc}
1+\cos \delta^{*}(\omega) & -i \sin \delta^{*}(\omega) \\
i \sin \delta^{*}(\omega) & 1-\cos \delta^{*}(\omega)
\end{array}\right)
\end{aligned}
$$

where we have introduced the Onsager hyperbolic triangle, shown in Fig. 2 [1].

The transfer matrix $V^{(b)}$ has angle $\delta_{1}^{*}(\omega)$ in place of $\delta^{*}$. The value of this is that we can use the hyperbolic generalizations of the al-Kashi triangle formulas as a common vehicle for algebraic simplification in a systematic way.

We are now almost in a position to state the results for the problem considered. The problem stated is a four-point function, in the language of (19), that may be reduced to a double sum by use of the generalized Wick's theorem noted above. We may extract the relevant terms by first discarding any $n$-independent terms, typically corresponding to droplet states that do not cross the strip, and then discarding any $k$-dependent terms which correspond to cross-connected bubbles, not the single configurations that interest us. In effect this calculation immediately distinguishes between three

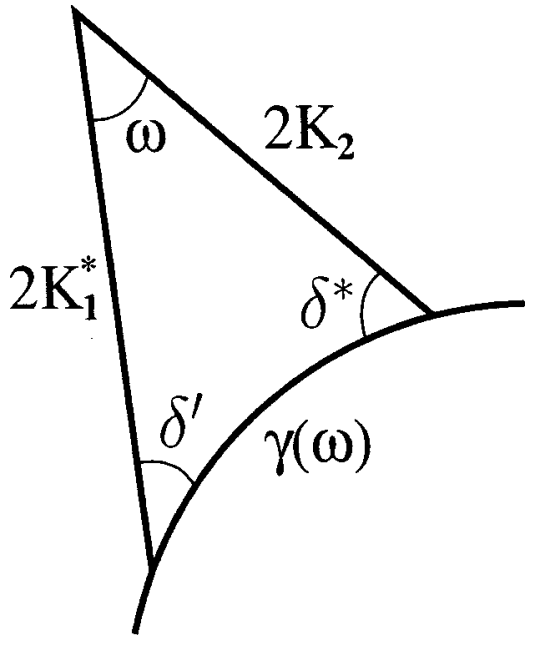

FIG. 2. Onsager's hyperbolic triangle. See text for details.

identifiable alternative configurations in the double system; two bubbles on the respective walls, two interfaces (Fig. 1), and cross-connected interfaces which are less easily visualized. Our selection is merely an expression that we wish to make $k \gg n$ implicitly forming a double copy of two otherwise uncoupled antiperiodic systems. It is important that we take these limits, $M \rightarrow \infty$ then $k \rightarrow \infty$, in sequence so that the configurations are identifiable. This thus defines and justifies the limiting process introduced in Eq. (2).

The final expression is the product of two identical integrals where the element associated with a single interface is

$$
Z(N, \infty ; b, \phi)=\frac{1}{2 \pi} \int_{-\pi}^{\pi} \frac{e^{i n \omega}}{A_{N}(\omega)} d \omega
$$

where the term in the denominator emerges from the sequence of transformations above Eq. (30). With the notation developed in Eq. (23) we find

$$
A_{\infty}(\omega)=\frac{e^{2 N \gamma(\omega)} e^{-2 K_{2}}\left(1+\cos \delta^{*}\right)\left(e^{\gamma(\omega)}-\lambda_{-}\right)\left(e^{\gamma(\omega)}-\lambda_{+}\right)}{4 \sinh 2 b K_{1} \sinh 2 K_{1}^{*} \sinh \gamma(\omega)}
$$

in the limit described by Eq. (32). For finite $N$, we may write $A_{N}(\omega)$ as a sum of three exponentially different terms,

$$
A_{N}(\omega)=p(\omega) e^{2 N \gamma(\omega)}-q(\omega)+r(\omega) e^{-2 N \gamma(\omega)},
$$

where $p(\omega) e^{2 N \gamma(\omega)} \equiv A_{\infty}(\omega)$. The analysis of Eq. (35) and its consequences for the finite size effects of the phase boundary of the GZZ transition will appear elsewhere [19]; here, however, we consider Eq. (34).

The terms $\lambda_{ \pm}$are roots of a quadratic term and are given by

$$
\lambda_{ \pm}=\mathrm{w} \pm \sqrt{\mathrm{w}^{2}-\frac{2 \mathrm{w} \cosh 2 K_{1}^{*}}{\cosh 2 K_{2}}+1}
$$

where 


$$
\mathrm{w}=\cosh 2 K_{2}\left(\cosh 2 K_{1}^{*}-\sinh 2 K_{1}^{*} \cosh 2 b K_{1}\right),
$$

which is analogous to a similar result for wetting at a wall [22]. The Onsager function $\gamma(\omega)$ [1] is defined by

$$
\cosh \gamma(\omega)=\cosh 2 K_{1}^{*} \cosh 2 K_{2}-\sinh 2 K_{2} \sinh 2 K_{1}^{*} \cos \omega,
$$

the necessary features of which are discussed below.

The integral (33) can be evaluated by contour techniques, and so we must choose an appropriate path within the $\omega$ plane. The Onsager function $\gamma(\omega)$ is $2 \pi$ periodic and has branch cuts in both the lower and upper half planes which extend from $2 i\left(K_{1}-K_{2}^{*}\right)$ to $2 i\left(K_{1}+K_{2}^{*}\right)$ and the negative of this respectively. It has been long established that these cuts provide information regarding the bulk properties of the model $[20,21]$. The factor $e^{i n \omega}$ in Eq. (33) ensures not only the convergence of the integral, but in the limit $n$ large that we can associate the leading order behavior of an element with the feature in the complex $\omega$ plane that lies closest to the real line. These features invariably lie on the imaginary axis to ensure the nonoscillatory behavior of any correlation lengths.

Examination of Eq. (33) reveals that for this problem we have three competing terms. The lower branch point from the Onsager function (contributing bulk terms), any zeros of $A_{N}(\omega)$ (contributing pinned terms), and a saddle point coming from the exponentiated term (contributing terms representing an interface at a constant angle). This last term has been used in previous calculations to determine the angular dependent surface tension in the Ising lattice [13]. In this problem we shall ignore the bulk contribution as this does not provide any leading order behavior. This situation is depicted in Fig. 3. The leading order terms are thus given by

$$
\gamma^{\prime}\left(i \nu_{0}\right)=\tan \phi
$$

which corresponds to the saddle point path and

$$
e^{\gamma\left(i \nu_{+}\right)}=\lambda_{+}
$$

which corresponds to the relevant pole in the upper half plane (we select the + root as this is the pole that is below the lower branch point). The transition thus takes place when these two features cross in the complex $\omega$ plane and we can therefore determine the phase boundary for this transition by equating $\nu_{0}$ and $\nu_{+}$. This mechanism for a phase transition



FIG. 3. (Color online) A plot showing the variation of the features in the complex plane as temperature varies. The only part of the plane that is plotted is the imaginary axis and the inverse temperature $\beta$ is shown on the horizontal axis. The lower branch point (bulk behavior) is shown in black and thus its intercept with the horizontal axis defines the bulk critical temperature. Below the feature are plotted the saddle point path [dark gray (blue)] and the pole [light gray (red)] whose behavior is described in the text. The system shown is an isotropic system with $\phi=75^{\circ}, b=0.6$.

has not to our knowledge been previously reported.

We can demonstrate the calculation very simply in the special case $\phi=\pi / 4$ on the isotropic lattice $\left(K_{1}=K_{2} \equiv K\right)$. We find

$$
\begin{aligned}
\cosh \nu_{0} & =\frac{1}{2}\left(\frac{1}{\sinh 2 K}+\sinh 2 K\right) \\
& =\frac{\cosh ^{2} 2 K}{\sinh 2 K}-\frac{1}{2}\left(\frac{1}{\lambda_{+}}+\lambda_{+}\right) \\
& =\cosh \nu_{+}
\end{aligned}
$$

which yields the simple solution $\lambda_{+}=\sinh 2 K$ (care must be taken in selecting the correct root). By using the definition of both $\lambda_{+}$and $w$ we find the concise solution for the phase boundary. This is most easily expressed as a critical value for the weakening parameter $b$ as

$$
b^{*}=\frac{1}{2 K} \ln (\cosh 2 K) .
$$

The calculation for general angle in an isotropic lattice is more involved, though not technically difficult, and here we provide some algebraic guidelines. We find

$$
\cosh \gamma\left(i \nu_{0}\right)=-\frac{\cosh 2 K_{1}^{*} \cosh 2 K_{2} \pm \sqrt{\left(\tan ^{2} \phi-1\right)^{2}+\cosh ^{2} 2 K_{2} \cosh ^{2} 2 K_{1}^{*}+\left(\tan ^{2} \phi-1\right)\left(\cosh ^{2} 2 K_{2}+\cosh ^{2} 2 K_{1}^{*}\right)}}{\tan ^{2} \phi-1}
$$

and

$$
\cosh \gamma\left(i \nu_{+}\right)=\frac{\mathrm{w}^{2} \cosh 2 K_{1}^{*}-\left(\mathrm{w} \cosh 2 K_{1}^{*}-\cosh 2 K_{2}\right)\left(\mathrm{w}^{2}-2 \mathrm{w} \cosh 2 K_{1}^{*} / \cosh 2 K_{2}+1\right)^{1 / 2}}{2 \mathrm{w} \cosh 2 K_{1}^{*}-\cosh 2 K_{2}}
$$

which upon equating satisfyingly factorize to give a quadratic equation in the wetting parameter w: 


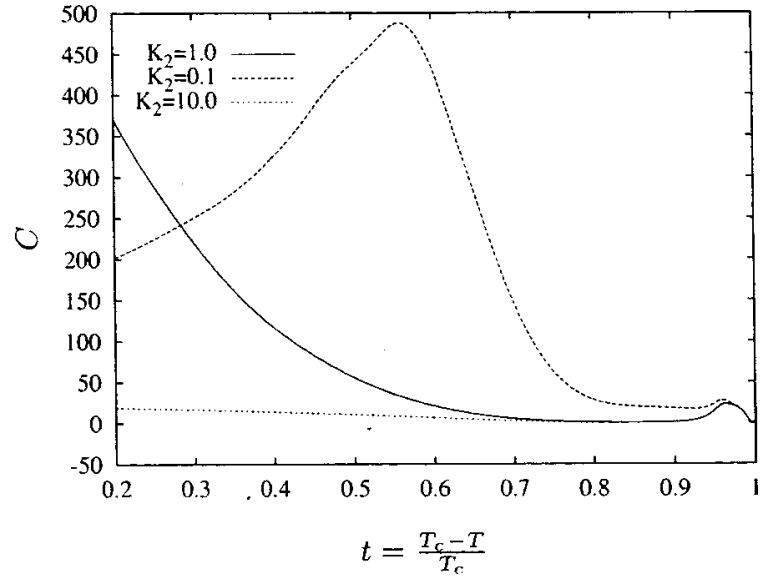

FIG. 4. Specific heat curves for $K_{2}=10.0,1.0,0.1$ show that the GZZ transition is independent of $K_{2}$ coupling strengths (the small peaks on the right). The bulk properties, however, change drastically, as they should; this is exemplified by the bulk transition peaks (the big ones on the left). The curves are results of Wang-Landau Monte Carlo simulations which provide data for continuous $t$; for details see [17].

$$
\mathrm{w}^{2}\left(\tan ^{2} \phi-1\right)+\cosh 2 K_{2}\left(2 \mathrm{w} \cosh 2 K_{1}^{*}-\cosh 2 K_{2}\right)=0 .
$$

The reader will note the surprising feature of this expression: it is a quadratic in $w / \cosh 2 K_{2}$. As w is only linearly dependent on $\cosh 2 K_{2}$ this implies the unanticipated result that the phase boundary will be independent of the horizontal, $K_{2}$, bond couplings. Such a result was also found in a Monte Carlo study, Fig. 4. Explicitly, by expanding Eq. (45) above, we find

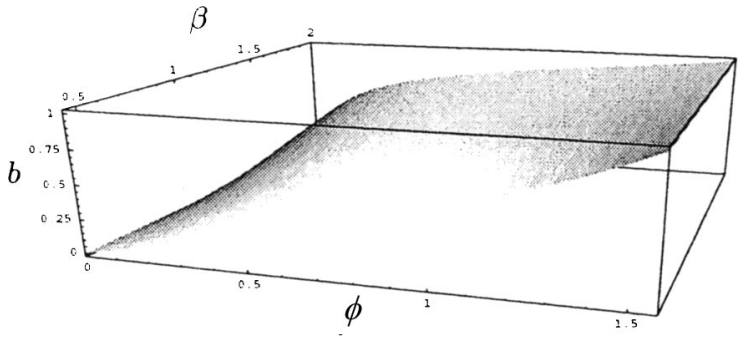

FIG. 5. A surface plot of the phase boundary in $\beta, \phi, b$ parameter space. Below the surface the interface will be pinned to the weakened boundary.

$$
b^{*}=\frac{1}{2 K_{1}} \ln \left(\frac{\tan \phi \cosh 2 K_{1}+\sqrt{1+\sinh ^{2} 2 K_{1} \tan ^{2} \phi}}{\tan \phi+1}\right)
$$

as the phase boundary. We emphasize that this equation represents a surface in the space of three parameters $b, \phi, T$ and hence there exist many trajectories in parameter space that will not exhibit a transition. Additionally, both $\nu_{0}$ and $\nu_{+}$ $\rightarrow 2 i\left(K_{1}-K_{2}^{*}\right)$ in the limit $T \rightarrow T_{\mathrm{c}}$, the bulk critical temperature, giving a consistent solution (this can be seen in Fig. 3). It is possible for this system to approach bulk criticality in either phase: this is not a mandatory precursor transition. The phase boundary surface defined by Eq. (46) is depicted for completeness in Fig. 5.

\section{ISING COMPUTATION II: MAGNETIZATION}

The assertions we have made about the nature of the GZZ phase transition in the preceding section were based on the exact analysis of the thermodynamics and some rather intuitive remarks about their interpretation. We now back these up with a discussion of the spatial dependence of the magnetization. This is given by

$$
\Sigma_{M}(r, s)=\frac{\left\langle\Phi_{+}^{0}\left|R(1, k)\left(V_{1} V_{2}\right)^{N-s} \sigma_{r}^{x}\left(V_{1} V_{2}\right)^{s} V_{b}\left(V_{2} V_{1}\right)^{N} R(n, n+k)\right| \Phi_{0}^{-}\right\rangle}{\left\langle\Phi_{+}^{0}\left|R(1, k)\left(V_{1} V_{2}\right)^{N} V_{b}\left(V_{2} V_{1}\right)^{N} R(n, n+k)\right| \Phi_{+}^{0}\right\rangle}
$$

at the point $(r, s)$.

Since we are interested in large $N$ asymptotics, after first taking $M \rightarrow \infty$, we will focus on the most significant terms in the spectral decomposition of $V^{N}$ and $V^{N-s}$ in the numerator, which are the two-particle ones.

Expressing $|+\rangle$ in terms of the $\left|\Phi_{ \pm}^{0}\right\rangle$ gives typically

$$
\begin{aligned}
& \frac{1}{4} \sum\left\langle\Phi_{+}^{0}\left|R(1, k) G^{\dagger}\left(-\beta_{0}\right) G\left(-\beta_{-1}\right)\right| \Phi_{+}\right\rangle e^{-N\left[\gamma\left(\beta_{0}\right)+\gamma\left(\beta_{-1}\right)\right]} \\
& \quad \times \sum_{j=0}^{\infty} \frac{1}{(2 j) !} \sum_{(\beta)_{2 j}}\left\langle\Phi_{+}\right| G\left(-\beta_{1}\right) G\left(-\beta_{0}\right) \tilde{V} G^{\dagger}\left(\beta_{1}\right) \cdots G^{\dagger}\left(\beta_{2 j}\right) \\
& \quad \times\left|\Phi_{+}\right\rangle \exp \left(-n \sum_{1}^{2 j} \gamma\left(\beta_{l}\right)\right) \times\left\langle\Phi_{+}\right| G\left(\beta_{2 j}\right) \cdots G\left(\beta_{1}\right) \sigma_{m}^{x} G^{\dagger}
\end{aligned}
$$

$$
\begin{aligned}
& \times\left(-\alpha_{0}\right) G^{\dagger}\left(-\alpha_{-1}\right)\left|\Phi_{-}\right\rangle \times\left\langle\Phi_{-}\right| G\left(-\alpha_{-1}\right) G\left(-\alpha_{0}\right) \\
& \times R(n, n+k)\left|\Phi_{-}^{0}\right\rangle e^{-(N-y)\left[\gamma\left(\alpha_{0}\right)+\gamma\left(\alpha_{-1}\right)\right]} .
\end{aligned}
$$

We use translational invariance (the reason for requiring cyclic symmetry) to move $\sigma_{r}^{x}$ to $\sigma_{1}^{x}=f_{1}^{\dagger}+f_{1}$, for which the central matrix element is known [23]. For large $n$, asymptotics will be obtained by taking $j=0$ and $j=2$. We take $M$ $\rightarrow \infty$, so that sums can be replaced by Riemann integrals; a little caution is needed here since we shall need the Cauchy principal part. We thus take $k \rightarrow \infty$, which separates the two interface lines by cyclic boundary conditions. With $r, s$ finite, we stay near the left hand inclined interface which will manifest the GZZ transition. This gives the results 


$$
\begin{aligned}
\Sigma_{M}(r, s) & \\
= & I_{N}^{-1} \frac{m^{*}}{\pi^{2}} \int_{-\pi}^{\pi} \int_{-\pi}^{\pi} \\
& \times \frac{e^{-(N-s) \gamma(\beta)} e^{-s \gamma(\alpha)} f_{+}\left(e^{i \beta}, e^{-i \alpha}\right) e^{-i n \alpha} e^{i(r-1 / 2)(\alpha-\beta)}}{\left[1+\cos \delta^{*}(\alpha)\right] A_{\infty}(\beta)} d \alpha d \beta,
\end{aligned}
$$

where

$$
I_{N}=\frac{1}{\pi} \int_{-\pi}^{\pi} d \omega \frac{e^{-i n \omega} e^{-N \gamma(\omega)}}{\left[1+\cos \delta^{*}(\omega)\right] A_{\infty}(\omega)}
$$

The exact details of $f_{+}\left(z_{1}, z_{2}\right)$ are not required; its only feature of relevance is the presence of a simple pole when $z_{1} z_{2}=1$ and the value of the residue at this point. We must therefore write Eq. (49) as a principal part integration, integrating $\beta$ (say) first and decompose the resulting integration with reference to the Plemelj formula. The $\beta$ integration represents configurations moving up from the lower boundary, which thus do not yet feel the influence of the grain boundary. Correspondingly the integration will be controlled by the saddle path from the boundary to the spin flip, given by

$$
(N-s) \gamma^{\prime}\left(i \nu_{\beta}\right)=i r .
$$

We find, in the limit $N, j$, and $n$ large,

$$
\Sigma_{M}(r, s) \sim m^{*}+\Gamma_{N}^{1} \frac{m^{*}}{\pi^{2}} \int_{-\infty}^{\infty} \int_{-\pi}^{\pi} \frac{e^{-(N-s) \gamma\left(i \nu_{\beta^{+}} \epsilon_{\beta}\right)} e^{-(N+s) \gamma(\alpha)} f_{+}\left(e^{i \beta}, e^{-i \alpha}\right) e^{-i(n-r) \alpha} e^{r \nu} \beta^{-i r \epsilon_{\beta}}}{\left[1+\cos \delta^{*}\left(i \nu_{\beta}+\epsilon_{\beta}\right)\right] p(\alpha)} d \alpha d \epsilon_{\beta} .
$$

Note that we have used the notation $p(\omega)$ defined in the text above so that we may write the exponentiated term in the denominator, making the saddle point interpretation more intuitive. The final integration, with respect to $\alpha$, may now be performed. This integration, now with the presence of the simple pole in the numerator, can have one of two behaviors, precisely analogous to that appearing in the first section of this article. The saddle is given by

$$
(N+s) \gamma^{\prime}\left(i \nu_{\alpha}\right)=i(n-r) .
$$

If the saddle $\nu_{\alpha}$ dominates then Eq. (52) becomes a double saddle integration. This is exactly the result found by previous authors [12] by making a simple change of variable. In terms of a new coordinate system $(x, y)$ defined along and normal to the mean geodesic configuration, these authors find the result is

$$
\begin{aligned}
m(x & \left.=\frac{1}{2} L \eta, y=\xi L^{\delta}\right) \\
& = \begin{cases}0, & 0<\delta< \\
m^{*} \operatorname{sgn}(\xi), & \delta>\frac{1}{2}, \\
m^{*} \operatorname{sgn}(\xi) \operatorname{erf}\left(|\xi|\left[\frac{2\left[\tau(\phi)+\tau^{\prime \prime}(\phi)\right]}{1-\eta^{2}}\right]^{1 / 2}\right) & \delta=\frac{1}{2},\end{cases}
\end{aligned}
$$

which we will comment upon shortly. Note that $L$ is defined by $L^{2}=(2 N+1)^{2}+n^{2}$.

For the pinned configuration, however, we find other results. First, we examine the behavior around the inclined section of interface. In a coordinate system defined analogously to the one above we find

$$
\begin{aligned}
m(x & \left.=\frac{1}{4} \mathcal{L} \eta, y=\xi \mathcal{L}^{\delta}\right) \\
& = \begin{cases}0, & 0<\delta<\frac{1}{2} \\
m^{*} \operatorname{sgn}(\xi), & \delta>\frac{1}{2}, \\
m^{*} \operatorname{sgn}(\xi) \operatorname{erf}\left(|\xi|\left[\frac{2\left[\tau(\phi)+\tau^{\prime \prime}(\phi)\right]}{1-\eta}\right]^{1 / 2}\right), & \delta=\frac{1}{2},\end{cases}
\end{aligned}
$$

a form almost identical to that above $\left(\mathcal{L}^{2} \equiv N^{2}+n^{2}\right)$. Note that in order to make a geometric interpretation of the pinned free energy per unit length, $\nu_{+}$, it is necessary to perform a short variational calculation; this is presented in the Appendix. We may also probe behavior close to the pinned defect line. Here we find a different result: the interface is sharp in the thermodynamic limit with

$$
m\left(x=n / 2 \eta, y=(N-\xi)^{\delta}\right)=m^{*} \operatorname{sgn}(\xi)
$$

independent of $\delta$.

\section{CONCLUSIONS}

In this article we have presented in detail the exact Ising computations pertaining to the geodesic to zigzag transition, that we have presented elsewhere. The exact calculation reveals a previously unreported mechanism for transitions of this type in the planar Ising model. Mathematically speaking this is a switch from a saddle dominated integral to a pole dominated integral representing the partition function ratio. Unlike in edge wetting [9] the bulk branch points play no role. It is interesting to note that the phase boundary is independent of one of the Ising couplings, $K_{2}$. 


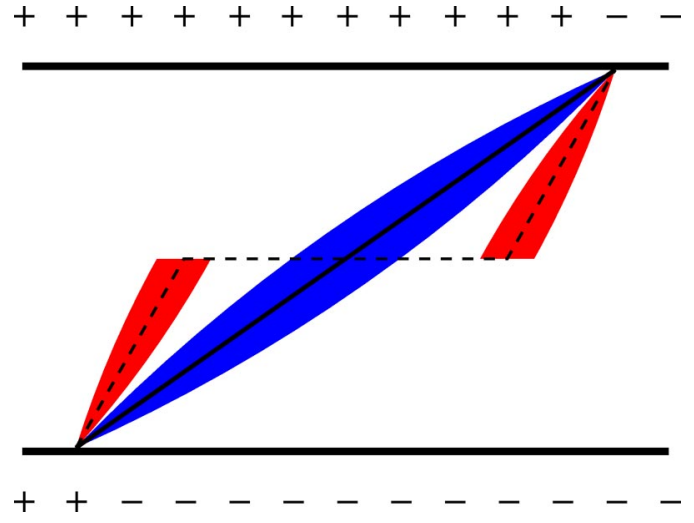

FIG. 6. (Color online) A cartoon showing the competing interfacial configurations with their respective fluctuation "envelopes." See text for further details.

To further justify this picture we have also presented in detail the derivation and results of a calculation for a magnetization using a movable "probe" spin. Such a calculation allows us to examine the geometric fluctuation picture in greater detail and confirm our intuitive picture. We may interpret the results presented above in the following way. In the geodesic case, as established exactly by Abraham and Upton, the interface undergoes fluctuations of the order of the square root of the total length of the interface. These fluctuations therefore diverge with the system size. In the zigzag case we have two distinct fluctuation phenomena occurring in the system at the different interface sections. The inclined interface sections, connecting the boundary to the defect line, also undergo square root fluctuations, but as the ends are not fixed the point where the interface meets the grain boundary will fluctuate considerably. The pinned section of interface is markedly different; its fluctuation behavior does not diverge with the system size and its width is strictly finite. In effect, in the pinned phase, the system behaves as a geodesic phase which has been sliced in half, linked by a horizontal, fluctuation-suppressed, section of interface (Fig. 6).

An alternative interpretation of the transition is by instead considering a critical angle, rather than a critical weakening. Rearranging Eq. (46) for $\phi^{*}$, the imposed angle at which the transition occurs for given $b$ and $T$, we find

$$
\tan \phi^{*}=\frac{1-e^{4 K_{1} b}}{\left(e^{2 K_{1} b}-e^{2 K_{1}}\right)\left(e^{2 K_{1} b}-e^{-2 K_{1}}\right)},
$$

an expression which respects the two known limits $b$ $=0 \Rightarrow \phi=0, b=1 \Rightarrow \phi=\pi / 2$. We may now write the transition in terms of this angle and its value relative to the contact angle. Expressed in this way this transition scenario becomes reminiscent of filling $[24,25]$. Additionally the fluctuation behavior of the vertical interface sections in the zigzag phase is reminiscent of the interpretation of filling in terms of "breather" modes.

\section{ACKNOWLEDGMENTS}

All the authors thank Professor K. Kaski for hospitality in Helsinki. D.B.A. thanks the Laboratory of Computational

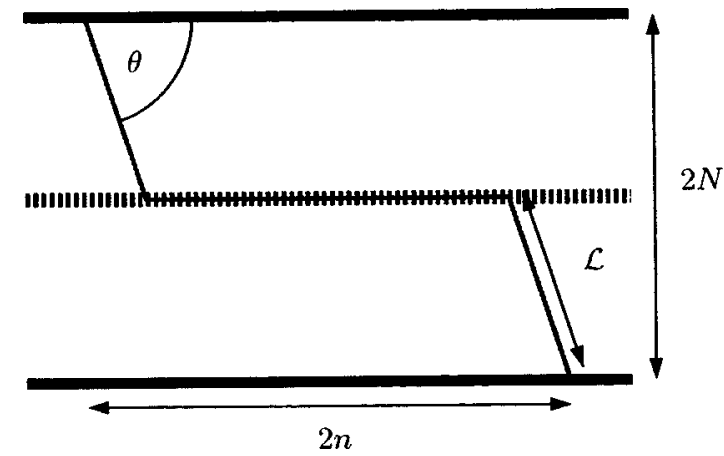

FIG. 7. (Color online) A simple picture defining the length scales used in the text.

Engineering at the Helsinki University of Technology (LCEHUT) for support. D.B.A. and A.J.W. acknowledge financial support from the EPSRC under Grants No. GR/M04426 and No. GR/R83712/01, respectively. V.M. was partially supported by the Academy of Finland, Research Centre for Computational Science and Engineering, Project No. 44897 (Finnish Centre of Excellence Program).

\section{APPENDIX: SIMPLE MINIMIZATION SCHEME}

We may find a geometrical interpretation for the quantities used above by following a simple minimization scheme, taking the values for the surface tension as those already computed within the Ising model. This is not, in any way, a substitute for an exact calculation, but is an effective way of interpreting thermodynamics and has been used with great success for understanding geometrical influence on interfacial configurations [25-27].

We begin by defining an energy function with the angular, symmetric inclination of the zigzag, $\theta$ say, as the controlling parameter. In terms of the notation defined in Fig. 7 we find

$$
f(\theta)=2 \tau_{A B}(\theta) \frac{N}{\sin \theta}+2 \tau_{D}[n-\mathcal{L} \cos (\theta)]
$$

where $\tau_{A B}$ and $\tau_{D}$ are the surface tensions associated with the inclined interface section and the defect line surface tension, respectively. The formula for $\tau_{A B}$ is now well established [12] and we quote

$$
\tau_{A B}(\theta)=\sin \theta \gamma\left[i \nu_{s}(\theta)\right]+\nu_{s}(\theta) \cos (\theta)
$$

where $\nu_{s}(\theta)$ is defined by

$$
\gamma^{\prime}\left[i \nu_{s}(\theta)\right]=i \cot \theta .
$$

We may thus perform the minimization by simple differentiation of Eq. (A1) with respect to $\theta$; we find

$$
f^{\prime}(\theta)=\frac{2 N}{\sin ^{2} \theta}\left[\tau_{D}-\nu_{s}(\theta)\right]
$$

and therefore at the minima we can associate the defect line tension $\tau_{D} \equiv \nu_{+}$with a well defined angle $\theta$. This association is required to define the coordinate system for the magnetization calculation and ensures the self-consistency of the methods used. 
[1] L. Onsager, Phys. Rev. 65, 117 (1944).

[2] B. Kaufman, Phys. Rev. 76, 1232 (1949).

[3] T. D. Schultz, D. C. Mattis, and E. H. Lieb, Rev. Mod. Phys. 36, 856 (1964).

[4] R. Evans, J. Phys.: Condens. Matter 2, 8089 (1990).

[5] M. E. Fisher and H. Nakanishi, J. Chem. Phys. 75, 5875 (1981).

[6] K. Binder et al., J. Stat. Phys. 110, 1411 (2003).

[7] A. O. Parry, A. J. Wood, C. Ráscon, J. Phys.: Condens. Matter 13, 4591 (2001).

[8] S. Dietrich, in Phase Transitions and Critical Phenomena, edited by C. Domb and J. L. Lebowitz (Academic, London, 1988), 12, p. 1; H. W. Diehl, in Phase Transitions and Critical Phenomena, edited by C. Domb and J. L. Lebowitz (Academic Press, London, 1986), Vol. 10, p. 76; D. B. Abraham, ibid.1.

[9] D. B. Abraham, Phys. Rev. Lett. 44, 1165 (1980).

[10] D. B. Abraham, J. Phys. A 14, L369 (1981).

[11] M. E. Fisher, J. Stat. Phys. 34, 667 (1984).

[12] D. B. Abraham and P. J. Upton, Phys. Rev. B 37, R3835 (1988).

[13] D. B. Abraham and L-F. Ko, Phys. Rev. Lett. 63, 275 (1989).

[14] D. B. Abraham, F. Latrémolière, and P. J. Upton, Phys. Rev. Lett. 71, 404 (1993).
[15] D. B. Abraham, V. Mustonen, and A. J. Wood, Phys. Rev. Lett. 93, 076101 (2004).

[16] D. B. Abraham, V. Mustonen, and A. J. Wood, J. Phys. A 37, L233 (2004).

[17] D. B. Abraham, V. Mustonen, and A. J. Wood Phys. Rev. E 70, 066138 (2004).

[18] N. N. Bogoliubov, Nuovo Cimento 7, 794 (1958); J. G. Valatin, Phys. Rev. 122, 1012 (1961).

[19] D. B. Abraham, V. Mustonen, and A. J. Wood (unpublished).

[20] T. T. Wu, Phys. Rev. 149, 380 (1966).

[21] L. P. Kadanoff, Phys. Rev. 188, 859 (1969).

[22] For wetting, the parameter is defined as $\mathrm{w}=e^{2 K_{2}}\left(\cosh 2 K_{1}^{*}\right.$ $\left.-\sinh 2 K_{1}^{*} \cosh 2 b K_{1}\right)$; see Ref. [9].

[23] D. B. Abraham, Commun. Math. Phys. 59, 17 (1978).

[24] A. O. Parry, C. Rascón, and A. J. Wood, Phys. Rev. Lett. 83, 5535 (1999); D. B. Abraham and A. Maciolek, ibid. 89, 286101 (2002).

[25] A. O. Parry, A. J. Wood, E. Carlon, and A. Drzewiński. Phys. Rev. Lett. 87, 196103 (2001).

[26] D. B. Abraham, V. Mustonen, and A. J. Wood, Europhys. Lett. 63, 408 (2003).

[27] D. B. Abraham, A. O. Parry, and A. J. Wood, Europhys. Lett. 60, 106 (2002). 